\title{
Clustering in complex networks. I. General formalism
}

\author{
M. Ángeles Serrano ${ }^{1}$ and Marián Boguñá ${ }^{2}$ \\ ${ }^{1}$ School of Informatics, Indiana University, Eigenmann Hall, 1900 East Tenth Street, Bloomington, Indiana 47406, USA \\ ${ }^{2}$ Departament de Física Fonamental, Universitat de Barcelona, Martí i Franquès 1, 08028 Barcelona, Spain
}

(Received 15 August 2006; revised manuscript received 11 October 2006; published 28 November 2006)

\begin{abstract}
We develop a full theoretical approach to clustering in complex networks. A key concept is introduced, the edge multiplicity, that measures the number of triangles passing through an edge. This quantity extends the clustering coefficient in that it involves the properties of two-and not just one-vertices. The formalism is completed with the definition of a three-vertex correlation function, which is the fundamental quantity describing the properties of clustered networks. The formalism suggests different metrics that are able to thoroughly characterize transitive relations. A rigorous analysis of several real networks, which makes use of this formalism and the metrics, is also provided. It is also found that clustered networks can be classified into two main groups: the weak and the strong transitivity classes. In the first class, edge multiplicity is small, with triangles being disjoint. In the second class, edge multiplicity is high and so triangles share many edges. As we shall see in the following paper, the class a network belongs to has strong implications in its percolation properties.
\end{abstract}

DOI: 10.1103/PhysRevE.74.056114

PACS number(s): 89.75.-k, 87.23.Ge, 05.70.Ln

\section{INTRODUCTION}

The important role of transitive relations in complex interaction systems has been exposed since the work of Georg Simmel, a popular 19th century German sociologist who pointed out the interest in triads in a pioneering work on the concept of social structure [1]. Simmel understood society as a web of patterned interactions and focused on the study of the forms of these interactions as they occur and reoccur in diverse historical periods and cultural settings. His emphasis on quantitative aspects lead him to analyze, in particular, dyadic versus triadic relationships, to find that when a dyad is formed into a triad, the apparently insignificant fact that one member has been added actually brings about a major qualitative change, various actions and processes becoming possible where previously they could not take place. The triad is then seen as the simplest structure in which the group as a whole can achieve domination over its component members, and so becomes the scenario exhibiting the simplest expression of the sociological drama.

In the study of complex networks, where large systems of interactions are mapped into comprehensible graphs [2-4], just vertices and edges are, nevertheless, usually recognized as the primary building blocks. Vertices represent the elementary units under mutual influence, and the interactions are modeled by edges linking them. Transitive relations, represented in this scheme by triangles, arise then as a secondary form of basic organization, made up of vertices connected by edges. However, the empirical evidence of a big number of triangles well above random expectations in the vast majority of real networks has brought this figure into attention, with a first reference to transitivity appearing in the literature of complex networks in the form of the clustering coefficient [5], a scalar measure quantifying the total number of triangles in a network through the average likelihood that two neighbors of a vertex are neighbors themselves. Triangles in complex networks are indissolubly tied to the analysis of degree correlations and they have been recognized as a fundamental element in the composition of recur- ring subgraphs, the so-called motifs [6], closely related to the large-scale organization of complex networks [7], their functionality or community structure $[8,9]$. So, in the framework of complex networks science, they have to be taken into account as a basic unbridged object, whose presence and self-organization can drastically impact network structure and properties.

In this paper and the following one, we develop a full theoretical approach to clustering in complex networks on the basis of former work [10,11]. It is extended and completed with interesting results previously unreported which lead to a substantially improved understanding of how clustering can be measured and which is the reach of its effects. In this paper, we begin by exposing in the next three sections the ways of measuring clustering at different depth levels. In Sec. II, we review, as a technical introduction and for completeness, the standard local and global measures related to one-vertex clustering. In Sec. III, we ask for the properties of not just one but two of the vertices involved in the triangles and to this end we review the concept of dyadic clustering from the definitions of edge multiplicity and edge clustering. Section IV treats the case of triadic clustering. In particular, we propose a different measure, the average nearest neighbors multiplicity $\bar{m}_{n n}(k)$, to compute triadic clustering in a practical way. In Sec. V, we explore the effects of degreedegree correlations on clustering. We find analytically that degree-degree correlations constrain the functional form of clustering and its maximum level. We also examine some empirical networks, finding a good agreement with our predictions. Section VI explores the condition for the simultaneous absence of degree correlation at the level of triangles and edges, which makes necessary the discrimination between weak and strong clustering. Finally, conclusions are drawn in Sec. VII. In this way, this first paper lays the general formalism. The following one will focus on percolation properties.

\section{ONE-VERTEX CLUSTERING}

In the context of complex networks, the concept of clustering was introduced as a way to quantify the transitivity of 
the connections. Several alternative definitions have been proposed, from global scalar quantities associated to the whole network $[12,13]$ to local measures describing the properties of single nodes. This is the case of the clustering coefficient first introduced by Watts and Strogatz [5],

$$
c_{i}=\frac{2 T_{i}}{k_{i}\left(k_{i}-1\right)},
$$

where $T_{i}$ is the number of triangles passing through vertex $i$ and $k_{i}$ is its degree. They also pointed out that real networks display a level of clustering-measured as the average of $c_{i}$ over the set of vertices in the network, the so-called clustering coefficient $C$ varying in the interval $[0,1]$-typically much larger than that produced by random effects.

The local clustering $c_{i}$ gives highly detailed information from a purely local perspective. One can adopt a compromise between the global property defined by $C$ and the full local information given by $c_{i}$ by defining an average of $c_{i}$ over the set of vertices of a given degree class [14], that is,

$$
\bar{c}(k)=\frac{1}{N_{k}} \sum_{i \in \Upsilon(k)} c_{i}=\frac{1}{k(k-1) N_{k}} \sum_{i \in \Upsilon(k)} 2 T_{i},
$$

where $N_{k}$ is the number of vertices of degree $k$ and $Y(k)$ is the set of such vertices. The corresponding scalar measure is called the mean clustering coefficient and can be computed on the basis of the degree distribution $P(k)$ as

$$
\bar{c}=\sum_{k} P(k) \bar{c}(k),
$$

which is related to the clustering coefficient as $C=\bar{c} /[1$ $-P(0)-P(1)]$. In fact, we have implicitly assumed that $\bar{c}(k$ $=0)=\bar{c}(k=1)=0$, whereas in the definition of $C$ we only consider an average over the set of vertices with degree $k>1$. This fact explains the difference between both measures.

In the case of uncorrelated networks, $\bar{c}(k)$ is independent of $k$. Furthermore, all the measures collapse and reduce to $C$ $[4,15,16]$,

$$
\bar{c}(k)=C=\frac{1}{N} \frac{\left(\left\langle k^{2}\right\rangle-\langle k\rangle\right)^{2}}{\langle k\rangle^{3}}, \quad k>1 .
$$

Therefore a functional dependence of $\bar{c}(k)$ on the degree can be attributed to the presence of correlations. Indeed, it has been observed that many real networks exhibit a power-law behavior $\bar{c}(k) \sim k^{-\alpha}$, with typically $0 \leqslant \alpha \leqslant 1$. Hence the degree dependent clustering coefficient has been proposed as a measure of hierarchical organization and modularity in complex networks [17].

\section{DYADIC CLUSTERING}

The degree dependent clustering coefficient described in the previous section measures the transitivity of a vertex that participates in a triangle and, in this sense, it is a projection over one vertex of a structure that involves three vertices. Then, it is natural to ask for the properties of not just one but two of the vertices involved in the triangle or, equivalently, to ask for the properties of edges involved in triangles.
To do so, let us define the multiplicity of an edge $m_{i j}$ as the number of triangles in which the edge connecting vertices $i$ and $j$ participates. This quantity is the analog for edges to the number of triangles attached to a vertex $T_{i}$. The two quantities are related through the trivial identity

$$
\sum_{j} m_{i j} a_{i j}=2 T_{i},
$$

which is valid for any network configuration. The matrix $a_{i j}$ is the adjacency matrix, giving the value 1 if there is an edge between vertices $i$ and $j$ and 0 otherwise.

Again, $m_{i j}$ is a local measure defined for every edge. We can coarse-grain and define the average multiplicity of the edges connecting the degree classes $k$ and $k^{\prime}, m_{k k^{\prime}}$, as

$$
m_{k k^{\prime}}=\frac{\sum_{i \in \Upsilon(k)} \sum_{j \in \Upsilon\left(k^{\prime}\right)} m_{i j} a_{i j}}{E_{k k^{\prime}}},
$$

where $E_{k k^{\prime}}$ stands for the number of edges between those degree classes (two times that number if $k=k^{\prime}$ ). The multiplicity matrix $m_{k k^{\prime}}$ is defined in the range $\left[0, m_{k k^{\prime}}^{c}\right]$, where $m_{k k^{\prime}}^{c}=\min \left(k, k^{\prime}\right)-1$ and it represents a measure of dyadic clustering that gives a more detailed description than $\bar{c}(k)$ on how triangles are shared among vertices of different degrees. Furthermore, as we shall see in the following paper, it contains relevant information to analyze the percolation properties of clustered networks.

Now, it is possible to find a relation between multiplicity and clustering. Taking into account the fact that the joint degree distribution can be defined as, with $E$ the total number of edges in the network, we obtain the following closure condition at the class level

$$
\sum_{k^{\prime}} m_{k k^{\prime}} P\left(k, k^{\prime}\right)=k(k-1) \bar{c}(k) \frac{P(k)}{\langle k\rangle} .
$$

Let us emphasize that this equation is an identity fulfilled by any network, which ties it with the degree detailed balance condition derived in [18]. These identities are important because, given their universal nature, they can be used to derive properties of networks regardless their specific details. As an example, in Ref. [19] the detailed balance condition was used to prove the absence of epidemic threshold in scale-free networks.

A global scalar measure can also be defined for dyadic clustering. It is the average multiplicity of the network, obtained by averaging $m_{k k^{\prime}}$ over all degree classes,

$$
\bar{m}=\sum_{k} \sum_{k^{\prime}} m_{k k^{\prime}} P\left(k, k^{\prime}\right)=\frac{\langle k(k-1) \bar{c}(k)\rangle}{\langle k\rangle} .
$$

Values of $\bar{m}$ close to zero mean that there are no triangles. When $\bar{m} \lesssim 1$, triangles are mostly disjoint and their number can be approximated as $T(k) \lesssim k / 2$. Otherwise, when $\bar{m} \gg 1$, triangles jam into edges, with many triangles sharing the same edge. Table I shows empirical values for the average multiplicity $\bar{m}$, the maximum multiplicity $m_{\max }$, and the clustering coefficient $C$ for different real networks. These are the 
TABLE I. Empirical values of the average multiplicity $\bar{m}$, the maximum multiplicity $m_{\max }$, and the clustering coefficient $C$ for different real networks.

\begin{tabular}{lccc}
\hline \hline Network & $\bar{m}$ & $m_{\max }$ & $C$ \\
\hline PIN & 0.30 & 10 & 0.12 \\
AS & 2.55 & 537 & 0.45 \\
PGP & 3.31 & 94 & 0.50 \\
Email & 3.90 & 28 & 0.27 \\
Co-authors & 4.22 & 74 & 0.74 \\
WTW & 27.13 & 163 & 0.66 \\
\hline \hline
\end{tabular}

Internet at the autonomous system level (AS) [20], the protein interaction network of the yeast $S$. Cerevisiae (PIN) [21], an intra-university email network [22], the web of trust of the pretty-good-privacy algorithm (PGP) for encrypted communications [23], the network of co-authorship among academics $[24,25]$, and the world trade web (WTW) of trade relationships among countries [26]. In all cases, except for the PIN network, the value of $\bar{m}$ indicates a noticeable jamming of triangles into edges.

An alternative way to quantify dyadic clustering is by means of the edge clustering coefficient, defined in [8] as

$$
\bar{c}\left(k, k^{\prime}\right)=\frac{m_{k k^{\prime}}}{m_{k k^{\prime}}^{c}} .
$$

The advantage of using the normalized version $\bar{c}\left(k, k^{\prime}\right)$ instead of $m_{k k^{\prime}}$ is that the edge clustering coefficient admits a probabilistic interpretation. Indeed, the one-vertex clustering coefficient $\bar{c}(k)$ can be viewed as the probability that two neighbors of a vertex of degree $k$ are connected. $\bar{c}\left(k, k^{\prime}\right)$ can in its place be interpreted as the probability that an edge connecting two vertices of degrees $k$ and $k^{\prime}$ share a common neighbor.

\section{TRIADIC CLUSTERING}

Clustering is a measure of three point correlations, although it is not evident from the definitions of one-vertex clustering and dyadic clustering, respectively calculated as $\bar{c}(k)$ and $m_{k k^{\prime}}$. To clarify this point, we use a similar approach to the one followed when analyzing two-point correlations. In that case, we made use of the matrix $E_{k k^{\prime}}$, which counts the number of edges among different degree classes, to define the joint degree distribution $P\left(k, k^{\prime}\right)$ giving information on the probability that a randomly chosen edge of the network is connecting two vertices of degrees $k$ and $k^{\prime}$. In the case of triadic clustering, the fundamental object is not any more the edge but the triangle itself. Thus let us define a completely symmetric tensor $T_{k k^{\prime} k^{\prime \prime}}$, which measures the number of triangles connecting vertices of the degree classes $k, k^{\prime}$ and $k^{\prime \prime}$ when $k \neq k^{\prime} \neq k^{\prime \prime}$, two times the number of triangles when two of the indices are equal, and six times the number of triangles when the three indices are equal. This tensor satisfies the following identity:

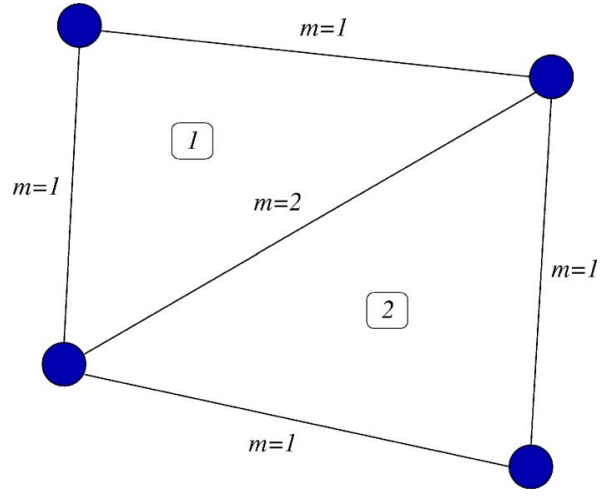

FIG. 1. (Color online) This figure illustrates the different information encoded in the functions $P\left(k, k^{\prime}\right)$ and $Q\left(k, k^{\prime}\right)$. In this simple graph, there are two kind of vertices, two of them with degree $k$ $=2$ and the other two of degree $k=3$. Each edge is labeled with its multiplicity. The function $\left(2-\delta_{k, k^{\prime}}\right) P\left(k, k^{\prime}\right)$ tells us which is the probability that a randomly chosen edge connects two vertices of degrees $k$ and $k^{\prime}$, respectively. Then, the probability that a randomly chosen edge connects two vertices of degrees $k=2$ and $k=3$ is $4 / 5$ and the probability of connecting two vertices of degree $k=3$ is $1 / 5$. In the case of $Q\left(k, k^{\prime}\right)$, we first have to chose randomly a triangleeither triangle labeled 1 or 2 in this example - and from this triangle one of its edges is randomly chosen. Using this procedure, the probability that an edge connects two vertices of degrees $k=2$ and $k$ $=3$ is $2 / 3$ that corresponds to first chose one triangle-with probability $1 / 2$ each in this particular graph-and then one edge-with probability $1 / 3$. Analogously, the probability of connecting two vertices of degree $k=3$ is $1 / 3$. Then we can write that $P(2,3)$ $=P(3,2)=2 / 5, P(3,3)=1 / 5$ and $Q(2,3)=Q(3,2)=2 / 6, Q(3,3)$ $=2 / 6$.

$$
\sum_{k^{\prime}} \sum_{k^{\prime \prime}} T_{k k^{\prime} k^{\prime \prime}}=\sum_{i \in \Upsilon(k)} 2 T_{i}=k(k-1) \bar{c}(k) P(k) N
$$

Then, we can define a joint distribution

$$
Q\left(k, k^{\prime}, k^{\prime \prime}\right) \equiv \frac{T_{k k^{\prime} k^{\prime \prime}}}{\langle k\rangle \bar{m} N}
$$

which measures the probability that a randomly chosen triangle connects three vertices of degrees $k, k^{\prime}$, and $k^{\prime \prime}$. The one point marginal distribution is in this case

$$
Q(k)=\sum_{k^{\prime}} \sum_{k^{\prime \prime}} Q\left(k, k^{\prime}, k^{\prime}\right)=\frac{k(k-1) \bar{c}(k) P(k)}{\langle k\rangle \bar{m}} .
$$

The two-point marginal distribution $Q\left(k, k^{\prime}\right)$ $=\sum_{k^{\prime \prime}} Q\left(k, k^{\prime}, k^{\prime \prime}\right)$ has an interesting interpretation. Indeed, it measures properties of the degrees of connected vertices and, in this sense, it is similar to $P\left(k, k^{\prime}\right)$. The main difference between both distributions is the way in which edges are selected. In the case of $P\left(k, k^{\prime}\right)$, an edge is randomly chosen and then one asks for the degrees at the ends of such edge. This selection mechanism implies that all edges in the network have the same probability to be chosen. In the case of $Q\left(k, k^{\prime}\right)$, one first selects a triangle with uniform probability among all the triangles present in the network and, once the triangle has been selected, one of its edges is randomly cho- 

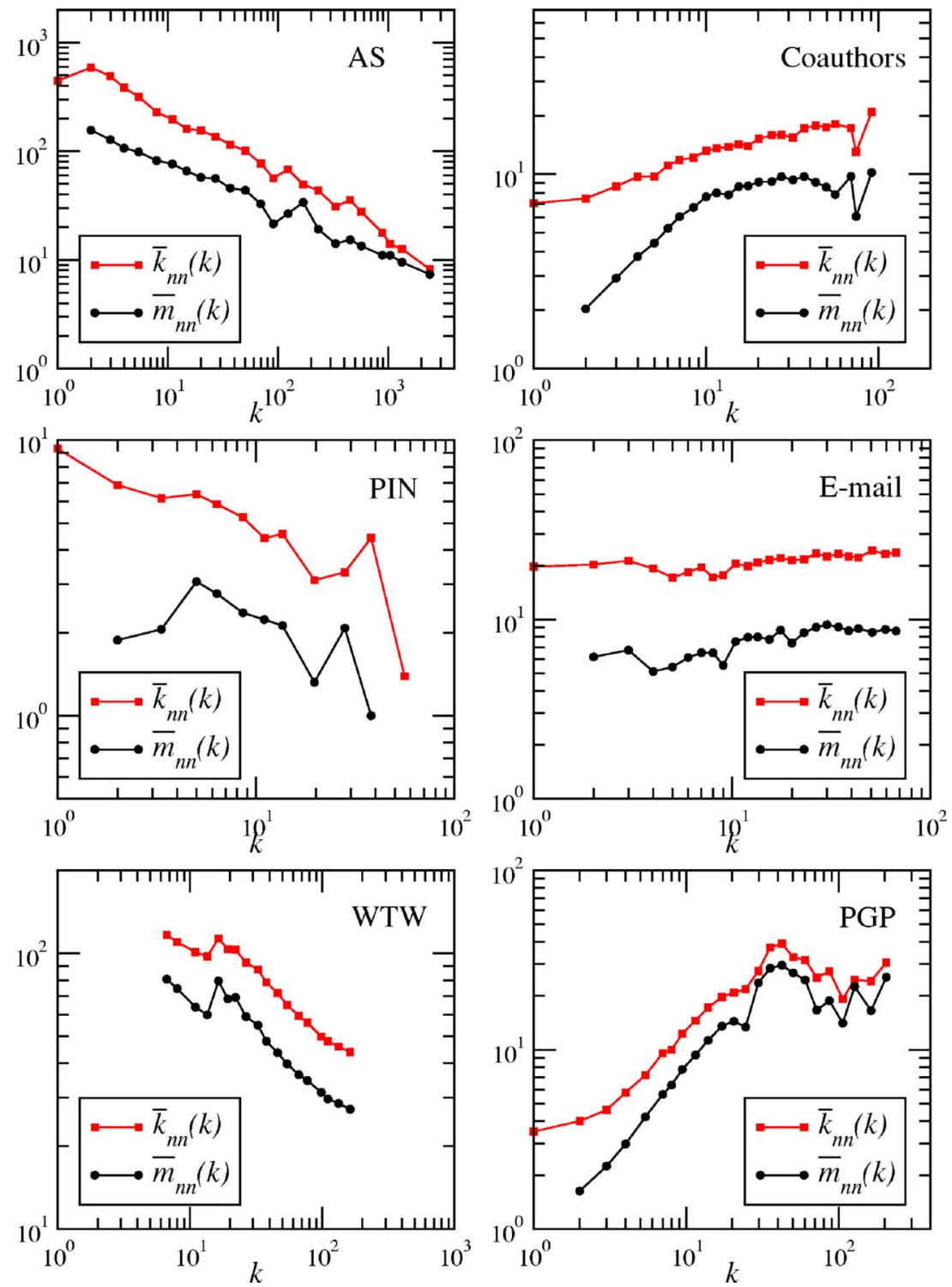

FIG. 2. (Color online) Empirical measures of the average nearest neighbors multiplicity as a function of $k$ compared with the average nearest neighbors degree.

sen. Then, the degrees of the vertices attached to this edge are measured. If one edge is shared by more than one triangle, this edge will be selected more often than edges that do not participate in triangles or in just one triangle. This implies that, in this case, edges are chosen with a nonuniform probability which is proportional to their multiplicity (Fig. 1 sketches this selection mechanism). This allows us to write

$$
Q\left(k, k^{\prime}\right)=\frac{m_{k k^{\prime}} P\left(k, k^{\prime}\right)}{\bar{m}} .
$$

Equations (12) and (13) eventually complete the fundamental functions which describes transitivity properties in complex networks. Indeed, being clustering a property that involves three distinct vertices, the most complete description is given by the function $Q\left(k, k^{\prime}, k^{\prime \prime}\right)$ which refers to triadic clustering. Nevertheless, when no that much information is required, we can work with the two-vertices marginal distribution $Q\left(k, k^{\prime}\right)$. However, by doing so, a new quantity encoding the kernel of dyadic clustering, the multiplicity $m_{k k^{\prime}}$, naturally appears accounting for the fact that edges can participate in more than one triangle. If we are interested in single vertices only, we are lead to the one-vertex marginal distribution $Q(k)$ which, again, introduces in a natural way the concept of clustering coefficient $c(k)$. All this means that, in fact, the functions $c(k)$ and $m_{k k^{\prime}}$ are just projections of the same fundamental object, described by $Q\left(k, k^{\prime}, k^{\prime \prime}\right)$.

Dealing in practice with the three-variables function $Q\left(k, k^{\prime}, k^{\prime \prime}\right)$ when studying triadic clustering is a rather complex task. A practical way to quantify triadic clustering requires the introduction of a different measure. To this end we propose to quantify the average multiplicity of edges among nearest neighbors in triangles attached to a vertex of degree $k$, which we call average nearest neighbors multiplicity $\bar{m}_{n n}(k)$ by analogy with the average nearest neighbors de- 
gree, the function $\bar{k}_{n n}(k)$ [27]. To compute $\bar{m}_{n n}(k)$ in a formal way, we first define the transition probability

$$
Q\left(k^{\prime}, k^{\prime \prime} \mid k\right)=\frac{\langle k\rangle \bar{m} Q\left(k, k^{\prime}, k^{\prime \prime}\right)}{k(k-1) \bar{c}(k) P(k)},
$$

from where we can write

$$
\bar{m}_{n n}(k)=\sum_{k^{\prime}, k^{\prime \prime}} m_{k^{\prime} k^{\prime \prime}} Q\left(k^{\prime}, k^{\prime \prime} \mid k\right) .
$$

As in the case of $\bar{k}_{n n}(k)$, in absence of correlations among the degrees of vertices forming triangles, the function $Q\left(k^{\prime}, k^{\prime \prime} \mid k\right)$ is independent of $k$, and so will be the case for the average nearest neighbors multiplicity. Therefore any nontrivial dependence of $\bar{m}_{n n}(k)$ on $k$ will signal the presence of correlations between the three degrees of the nodes that form triangles.

In Fig. 2, we show measures of this function for the different real networks analyzed through the paper. As one can see, the patterns follow closely those for the average nearest neighbors degree, that is, networks with assortative degree mixing also show an increasing $\bar{m}_{n n}(k)$, whereas disassortative ones show decreasing dependencies as a function of $k$. This can be intuitively understood if we consider that the function $\bar{k}_{n n}(k)$ appears to be an upper bound of $\bar{m}_{n n}(k)$. Despite this similarity, we also find differences in the behavior of $\bar{m}_{n n}(k)$ as compared to $\bar{k}_{n n}(k)$. In the case of the Internet at the autonomous system level, we find that both functions follow a power law decay as a function of $k$ but clearly with different exponents. In the case of the protein interaction network, $\bar{m}_{n n}(k)$ is approximately constant whereas $\bar{k}_{n n}(k)$ is a decreasing function of $k$.

\section{EFFECTS OF DEGREE-DEGREE CORRELATIONS ON CLUSTERING}

Degree-degree correlations constrain the maximum level of clustering a network can reach. A naive explanation for this is that, if the neighbors of a given node all have a small degree, the number of connected neighbors (and hence the clustering of such a node) will be bounded. This is the main idea behind the new measure of clustering introduced in [28]. However, we can make a step forward and analytically quantify this effect. The key point is to realize that the multiplicity matrix satisfies the inequality

$$
m_{k k^{\prime}} \leqslant \min \left(k, k^{\prime}\right)-1 \text {, }
$$

which comes from the fact that the degrees of the nodes at the ends of an edge determine the maximum number of triangles this edge can hold (see top plot in Fig. 3). Multiplying this inequality by $P\left(k, k^{\prime}\right)$ and summing over $k^{\prime}$ we get

$$
k(k-1) \bar{c}(k) \frac{P(k)}{\langle k\rangle} \leqslant \sum_{k^{\prime}} \min \left(k, k^{\prime}\right) P\left(k, k^{\prime}\right)-\frac{k P(k)}{\langle k\rangle},
$$

where we have used the closure condition Eq. (7). This inequality, in turn, can be rewritten as
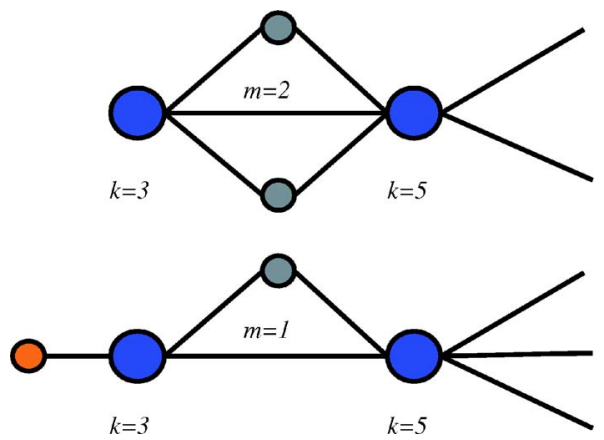

FIG. 3. (Color online) Sketch of the maximum possible value of the edge multiplicity in a case where the two vertices do not have neighbors of degree 1 (top) and where one of them has one of these neighbors (bottom). In the top plot, the blue vertices (large circles) have degrees $k=3$ and $k=5$ respectively and no neighbors of degree 1 . Then, the maximum value of $m$ for the edge connecting these two vertices is $m_{3,5}^{c}=\min (3-1,5-1)=2$. In the bottom plot, the vertex of degree $k=3$ has a neighbor of degree 1 . In this case, the maximum multiplicity for that edge is $m_{3,5}^{c}=\min (3-1-1,5-1)=1$.

$$
\bar{c}(k) \leqslant 1-\frac{1}{k-1} \sum_{k^{\prime}=1}^{k}\left(k-k^{\prime}\right) P\left(k^{\prime} \mid k\right) \equiv \lambda(k) .
$$

Notice that $\lambda(k)$ is always in the interval $[0,1]$ and therefore $\bar{c}(k)$ is always bounded by a function smaller (or equal) than 1 . In the limit of very large values of $k$, Eq. (18) reads

$$
\bar{c}(k) \leqslant \lambda(k) \approx \frac{\bar{k}_{n n}^{r}(k)-1}{k-1},
$$

where $\bar{k}_{n n}^{r}(k)$ is the average nearest neighbors degree of a vertex with degree $k$. The superscript $r$ (of reduced) refers to the fact that it is evaluated only up to $k$ and therefore $\bar{k}_{n n}^{r}(k) \leqslant k$. For strongly assortative networks $\bar{k}_{n n}^{r}(k) \sim k$, so that $\lambda(k) \sim \mathcal{O}(1)$ and there is no restriction in the decay of $\bar{c}(k)$. In the opposite case of disassortative networks, the sum term on the right hand side of Eq. (18) may be fairly large and then the clustering coefficient will have to decay accordingly.

It is important to mention that, although $\lambda(k)$ is an upper bound of $\bar{c}(k)$, it is not the lowest upper bound. In fact, in the inequality Eq. (16) we are not considering that the neighbors of the two vertices of degrees $k$ and $k^{\prime}$ might have not enough free connections. An obvious case corresponds to vertices of degree 1 . If the vertex of degree $k$ has $N(1 \mid k)$ neighbors of degree 1 (others than the one of degree $k^{\prime}$ ) and the one of degree $k^{\prime}$ has $N\left(1 \mid k^{\prime}\right)$ neighbors of degree 1 , then, the corrected inequality would be

$$
m_{k k^{\prime}} \leqslant \min \left[k-N(1 \mid k), k^{\prime}-N\left(1 \mid k^{\prime}\right)\right]-1 .
$$

This is sketched in Fig. 3. The problem is that, now, $N(1 \mid k)$ is a stochastic quantity with expected value $\langle N(1 \mid k)\rangle=(k$ -1) $P(1 \mid k)$ which, again, depends on the mixing properties of the network. This contribution is important in networks with a large number of vertices of degree 1 . 

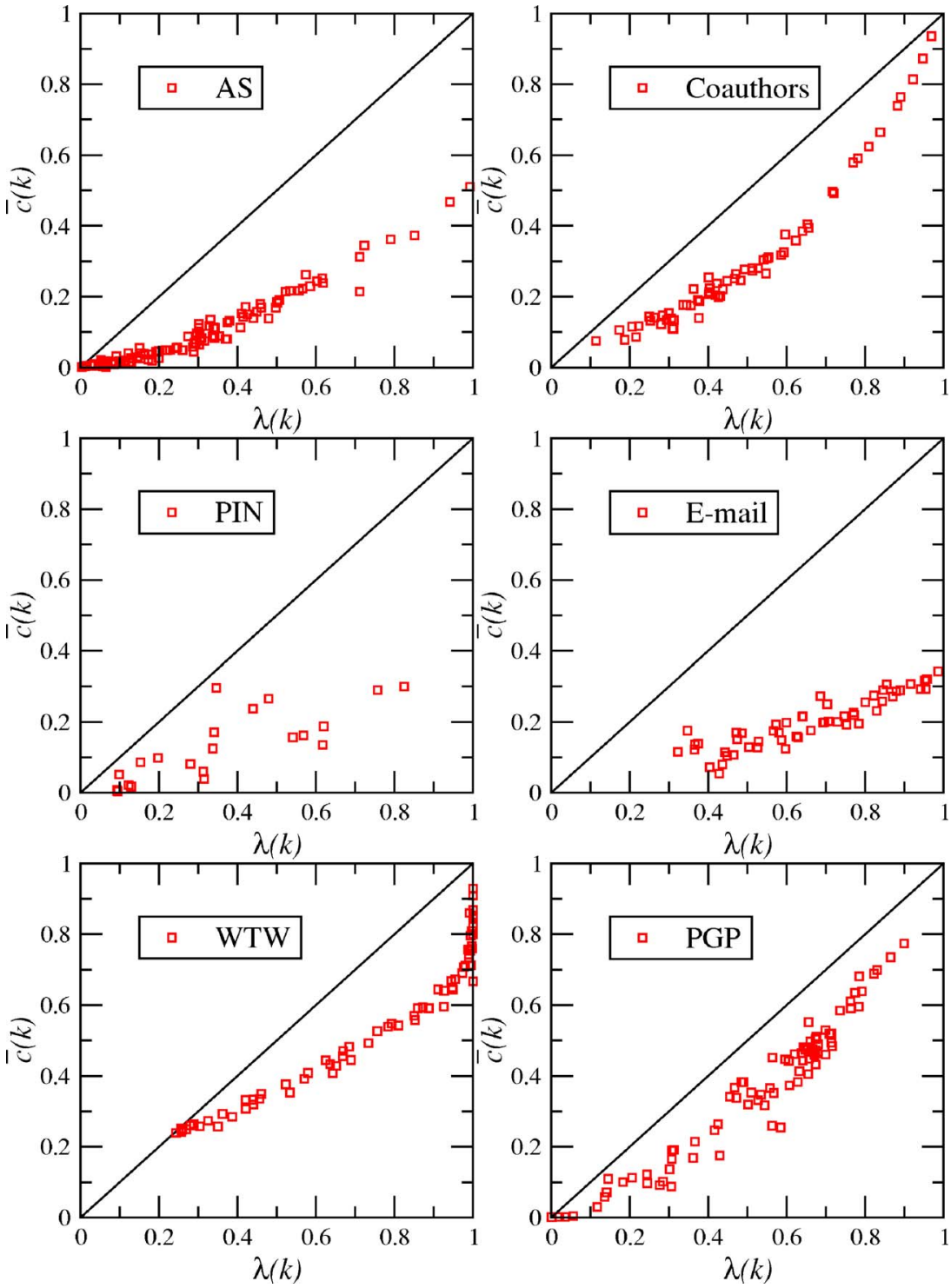

FIG. 4. (Color online) Clustering $\bar{c}(k)$ vs the maximum value $\lambda(k)$ for several real networks. In all cases, empirical measures fall below the diagonal line, validating the inequality Eq. (18).

The interplay between degree correlations and clustering can also be observed in real networks. We have measured the functions $\lambda(k)$ and $\bar{c}(k)$ for several empirical data sets, finding that the inequality Eq. (18) is always satisfied. In Fig. 4, we plot the clustering coefficient $\bar{c}(k)$ as a function of $\lambda(k)$. Each dot in these figures corresponds to a different degree class. As clearly seen, in all cases the empirical measures lie below the diagonal line, which indicates that the inequality Eq. (18) is always preserved. In Fig. 5, we show the ratio $\bar{c}(k) / \lambda(k)$. The rate of variation of this fraction is small and thus the degree dependent clustering coefficient can be computed as $\bar{c}(k)=\lambda(k) f(k)$, where $f(k)$ is a slowly varying function of $k$ that, in many cases, can be fitted by a logarithmic function. This result implies that, to a large extent, the functional dependence of $\bar{c}(k)$ is given by the particular shape of the degree-degree correlations. On the other hand, this also suggests that the edge clustering coefficient, given by Eq. (9), is also a weakly dependent-if not independent-

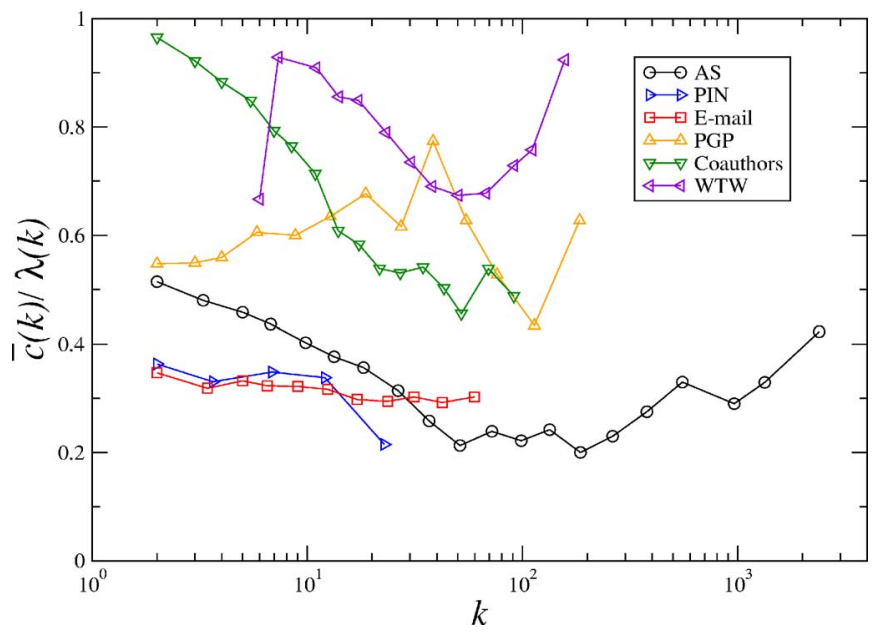

FIG. 5. (Color online) Empirical measures of the ratio between the clustering coefficient $\bar{c}(k)$ and the maximum value $\lambda(k)$ for different real networks. 

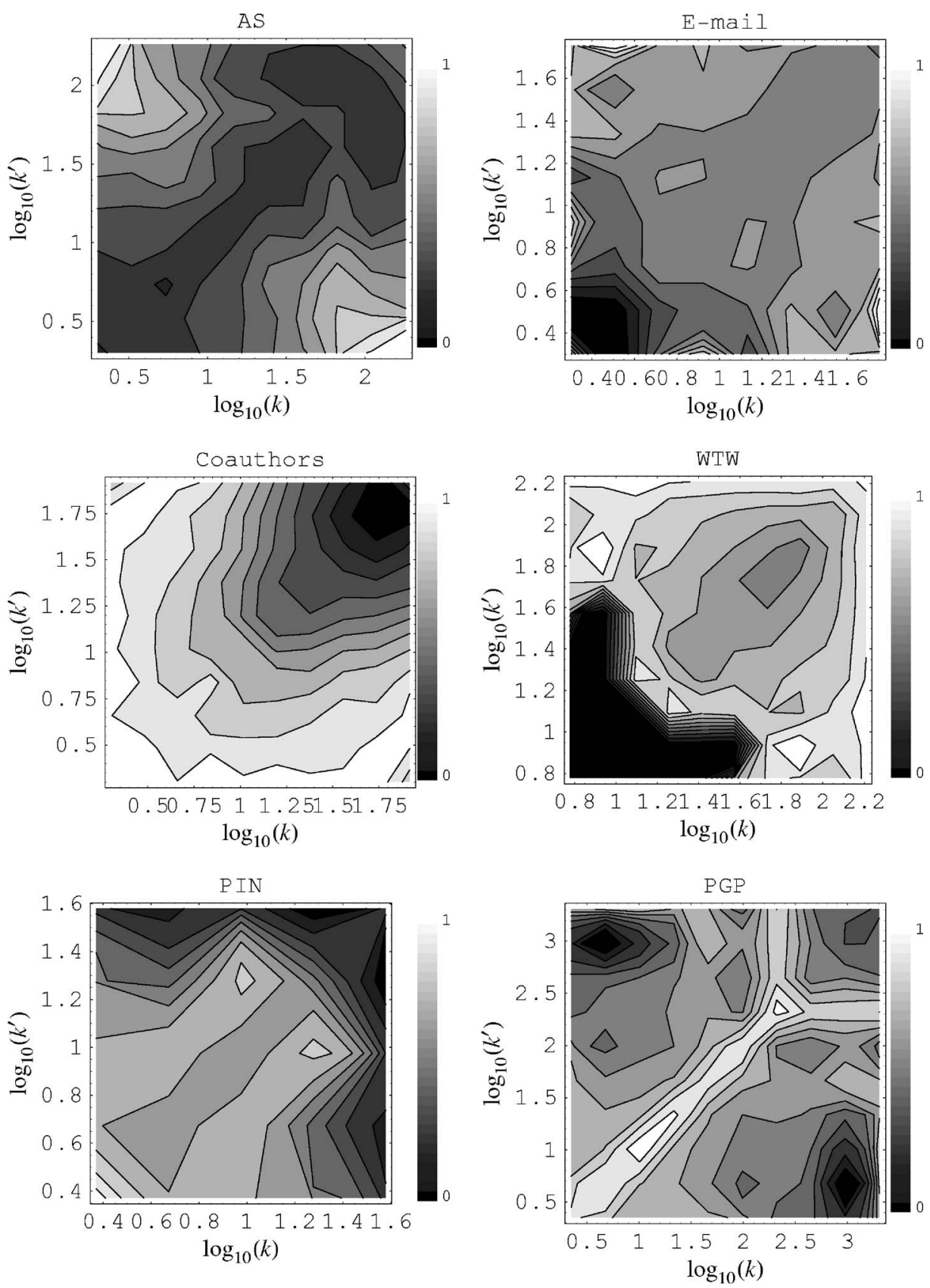

FIG. 6. Gray-scale contour plots of the edge clustering coefficient $\bar{c}\left(k, k^{\prime}\right)$ as a function of $k$ and $k^{\prime}$ for the different real networks analyzed.

function of the degrees $k$ and $k^{\prime}$. Indeed, empirical measures of $\bar{c}\left(k, k^{\prime}\right)$ in the studied real networks support this conjecture. Figure 6 shows contour plots of $\bar{c}\left(k, k^{\prime}\right)$ using a logarithmic binning of the axes. In all cases, there is a dominant intensity, which indicates that the edge clustering is approximately constant. As expected from the information shown in Fig. 5, the AS network and the co-author network are the less constant, although the variation of $\bar{c}\left(k, k^{\prime}\right)$ across different $\left(k, k^{\prime}\right)$ domains is not very pronounced. This result is particularly important because, unlike what happens for the degree dependent clustering coefficient $c(k)$, it allows to approximate dyadic clustering in many cases by a constant value.

\section{Scale-free networks}

Scale-free networks with degree distributions of the form $P(k) \sim k^{-\gamma}$ belong to a special class of networks which deserve a separate discussion. Indeed, it has been shown that, when the exponent of the degree distribution lies in the interval $\gamma \in(2,3]$ and its domain extends beyond values that scale as $N^{1 / 2}$, disassortative correlations are unavoidable for high degrees [29-32]. Almost all real scale-free networks fulfill these conditions and hence it is important to analyze how these negative correlations constrain the behavior of the clustering coefficient. Let us assume a power law decay of the average nearest neighbors degree of the form $\bar{k}_{n n}(k)$ 
$\sim \kappa k^{-\delta}$. One can prove that this function diverges in the limit of very large networks as $\bar{k}_{n n}(k) \sim\left\langle k^{2}\right\rangle \sim k_{c}^{3-\gamma}$, where $k_{c}$ is the maximum degree of the network [19]. Then, the prefactor $\kappa$ must scale in the same way which, in turn, implies that the reduced average nearest neighbors degree behaves as

$$
\bar{k}_{n n}^{r}(k) \sim k^{3-\gamma-\delta} .
$$

Then, from Eq. (19) the exponent of the degree dependent clustering coefficient $\alpha$ must verify the following inequality

$$
\alpha \geqslant \gamma+\delta-2 \text {. }
$$

Just as an example, in the case of the Internet at the autonomous system level [14], the reported values for these three exponents $(\alpha=0.75, \gamma=2.1$, and $\delta=0.5)$ satisfy this inequality close to the limit $(\alpha=0.75 \geqslant \gamma+\delta-2=0.6)$.

\section{UNCORRELATED NETWORKS AND THE DISTINCTION BETWEEN WEAK AND STRONG CLUSTERING}

When analyzing two-point correlations, the notion of uncorrelated network corresponds to a network in which the joint distribution $P\left(k, k^{\prime}\right)$ factorizes as

$$
P\left(k, k^{\prime}\right)=\frac{k k^{\prime} P(k) P\left(k^{\prime}\right)}{\langle k\rangle^{2}} .
$$

In the context of triangles, a network is uncorrelated when

$$
Q\left(k, k^{\prime}, k^{\prime \prime}\right)=Q(k) Q\left(k^{\prime}\right) Q\left(k^{\prime \prime}\right),
$$

where $Q(k)$ was given in Eq. (12). The question is whether functions $P\left(k, k^{\prime}\right)$ and $Q\left(k, k^{\prime}, k^{\prime \prime}\right)$ can factorize simultaneously. First, we restrict to the study of the factorization of $Q\left(k, k^{\prime}\right)$ instead of $Q\left(k, k^{\prime}, k^{\prime \prime}\right)$ since, due to the symmetric attribute as a tensor of the last function, the factorization of $Q\left(k, k^{\prime}\right)$ is a sufficient condition for the factorization of $Q\left(k, k^{\prime}, k^{\prime \prime}\right)$. Indeed, the function $Q\left(k, k^{\prime}\right)$ measures correlations between connected vertices when edges are weighted by their multiplicity, whereas $P\left(k, k^{\prime}\right)$ measures these correlations when edges are chosen with uniform probability. Given this difference in the selection mechanism of edges, $Q\left(k, k^{\prime}\right)$ and $P\left(k, k^{\prime}\right)$ cannot factorize simultaneously when the sample of edges is highly heterogeneous in their multiplicity values. In contrast, when $m_{i j}$ is either 0 or 1 , the sample of edges corresponding to triangles will become homogeneous and whenever $Q\left(k, k^{\prime}\right)$ factorizes, $P\left(k, k^{\prime}\right)$ factorizes too (for degrees larger than 1 ). In this case, we can write

$$
m_{k k^{\prime}} \propto(k-1)\left(k^{\prime}-1\right) \bar{c}(k) \bar{c}\left(k^{\prime}\right) .
$$

Since in this approach $m_{k k^{\prime}} \leqslant 1$ for $\forall k, k^{\prime}$, we have that

$$
\bar{c}(k) \leqslant \frac{1}{k-1}
$$

for any uncorrelated network at the two-vertex level. In other situations, one can construct uncorrelated networks at the level of triangles but, at the same time, there will appear some correlations at the level of edges and vice versa.
This suggests to partition the space of clustered networks into two main categories: weak transitivity - for networks with $\bar{c}(k) \leqslant(k-1)^{-1}, \forall k$ - and strong transitivity in the opposite case. As we will show in Paper II [33], the percolation properties of clustered networks are totally different depending on which one of these categories the network belongs to. This is related to the fact that, in the strong transitivity regime, the overlap of triangles is important, favoring thus the emergence of subgraphs which are tightly interconnected, the so-called $k$ cores [34]. In contrast, in the weak transitivity class, triangles are mostly disjoint and the topological properties of such networks are close to that of unclustered ones.

\section{CONCLUSIONS}

In this paper, we have provided a powerful formalism to understand transitive relations in complex networks. We have defined a fundamental quantity $Q\left(k, k^{\prime}, k^{\prime \prime}\right)$, which measures the probability that a randomly chosen triangle connects three vertices of degrees $k, k^{\prime}$, and $k^{\prime \prime}$. The summation over one variable of this fundamental distribution gives information about two of the vertices participating in the triangle and, in a natural way, introduces the multiplicity of edges among two classes of degrees $k$ and $k^{\prime}, m_{k k^{\prime}}$. The summation of $Q\left(k, k^{\prime}, k^{\prime \prime}\right)$ over two of its variables gives information about the properties of vertices that participate in triangles and, as in the previous case, naturally defines the degree-dependent clustering coefficient $\bar{c}(k)$. To quantify the extent of the correlations encoded in $Q\left(k, k^{\prime}, k^{\prime \prime}\right)$, we have proposed a different metric, the average nearest neighbors multiplicity $\bar{m}_{n n}(k)$, finding interesting patterns when measured in real networks. We have also found that, in real networks, the edge clustering coefficient, defined as the ratio between $m_{k k^{\prime}}$ and $\min \left(k-1, k^{\prime}-1\right)$, is a weakly dependent function of the degrees $k$ and $k^{\prime}$. This could serve as a basis for modeling of clustered networks. This result also suggest that the functional form of the degree-dependent clustering coefficient is mainly determined by the two-vertex correlation structure of the network. Last but not least, we have found the conditions for the simultaneous factorization of $Q\left(k, k^{\prime}, k^{\prime \prime}\right)$ and $P\left(k, k^{\prime}\right)$. This is only possible if $\bar{c}(k)<(k$ $-1)^{-1}$. This partitions the space of clustered networks into two main categories, networks with weak transitivity - those that satisfy $\bar{c}(k)<(k-1)^{-1}$ —and networks with strong transitivity - those that do not. In the first class, the multiplicity of edges is either 0 or 1 and triangles are disjoint. In the second class, edges are forced to share many triangles, giving rise to highly interconnected subgraphs. We shall see in Paper II how the class a network belongs to changes its percolation properties.

\section{ACKNOWLEDGMENTS}

This work has been partially supported by DGES, Grant No. FIS2004-05923-CO2-02 and Generalitat de Catalunya Grant No. SGR00889. M.B. thanks the School of Informatics at Indiana University, where part of this work was developed. 
[1] G. Simmel, Soziologie: Untersuchungen Uber Die Formen Der Vergesellschaftung (Duncket and Humbolt, Berlin, 1908).

[2] R. Albert and A.-L. Barabási, Rev. Mod. Phys. 74, 47 (2002).

[3] S. N. Dorogovtsev and J. F. F. Mendes, Evolution of Networks: From Biological Nets to the Internet and WWW (Oxford University Press, Oxford, 2003).

[4] M. E. J. Newman, SIAM Rev. 45, 167 (2003).

[5] D. J. Watts and S. H. Strogatz, Nature (London) 393, 440 (1998).

[6] R. Milo, S. Shen-Orr, S. Itzkovitz, N. Kashtan, D. Chklovskii, and U. Alon, Science 298, 824 (2002).

[7] A. Vázquez, R. Dobrin, D. Sergi, J.-P. Eckmann, Z. N. Oltvai, and A.-L. Barabási, Proc. Natl. Acad. Sci. U.S.A. 101, 17940 (2004).

[8] F. Radicchi, C. Castellano, F. Cecconi, V. Loreto, and D. Parisi, Proc. Natl. Acad. Sci. U.S.A. 101, 2658 (2004).

[9] G. Palla, I. Derényi, I. Farkas, and T. Vicsek, Nature 435, 814 (2005).

[10] M. A. Serrano and M. Boguñá, Phys. Rev. E 72, 036133 (2005).

[11] M. A. Serrano and M. Boguñá, Phys. Rev. Lett. 97, 088701 (2006).

[12] A. Barrat and M. Weigt, Eur. Phys. J. B 13, 547 (2000).

[13] M. E. J. Newman, S. H. Strogatz, and D. J. Watts, Phys. Rev. E 64, 026118 (2001)

[14] A. Vázquez, R. Pastor-Satorras, and A. Vespignani, Phys. Rev. E 65, 066130 (2002).

[15] M. Boguñá and R. Pastor-Satorras, Phys. Rev. E 68, 036112 (2003).

[16] Z. Burda, J. Jurkiewicz, and A. Krzywicki, Phys. Rev. E 70, 026106 (2004).

[17] E. Ravasz and A.-L. Barabási, Phys. Rev. E 67, 026112
(2003).

[18] M. Boguñá and R. Pastor-Satorras, Phys. Rev. E 66, 047104 (2002).

[19] M. Boguñá, R. Pastor-Satorras, and A. Vespignani, Phys. Rev. Lett. 90, 028701 (2003).

[20] R. Pastor-Satorras and A. Vespignani, Evolution and Structure of the Internet. A Statistical Physics Approach (Cambridge University Press, Cambridge, England, 2004).

[21] H. Jeong, S. P. Mason, A.-L. Barabási, and Z. N. Oltvai, Nature (London) 411, 41 (2001).

[22] R. Guimerà, L. Danon, A. Díaz-Guilera, F. Giralt, and A. Arenas, Phys. Rev. E 68, 065103(R) (2003).

[23] M. Boguñá, R. Pastor-Satorras, A. Díaz-Guilera, and A. Arenas, Phys. Rev. E 70, 056122 (2004).

[24] M. E. J. Newman, Phys. Rev. E 64, 016131 (2001).

[25] M. E. J. Newman, Phys. Rev. E 64, 016132 (2001).

[26] M. A. Serrano and M. Boguñá, Phys. Rev. E 68, 015101(R) (2003).

[27] R. Pastor-Satorras, A. Vázquez, and A. Vespignani, Phys. Rev. Lett. 87, 258701 (2001).

[28] S. N. Soffer and A. Vázquez, Phys. Rev. E 71, 057101 (2005).

[29] J. Park and M. E. J. Newman, Phys. Rev. E 68, 026112 (2003).

[30] Z. Burda and A. Krzywicki, Phys. Rev. E 67, 046118 (2003).

[31] M. Boguñá, R. Pastor-Satorras, and A. Vespignani, Eur. Phys. J. B 38, 205 (2004).

[32] M. Catanzaro, M. Boguñá, and R. Pastor-Satorras, Phys. Rev. E 71, 027103 (2005).

[33] M. Ángeles Serrano and M. Boguñá, Phys. Rev. E 74056115 (2006).

[34] S. N. Dorogovtsev, A. V. Goltsev, and J. F. F. Mendes, Phys. Rev. Lett. 96, 040601 (2006). 